\title{
Synthesis of novel 2-pyridyl- substituted 2,5-dihydro-2-imino- and 2-amino- furan derivatives via a three component condensation of alkyl isocyanides and acetylenic esters with di-(2-pyridyl) ketone or 2-pyridinecarboxaldehyde
}

\author{
Nourollah Hazeri, ${ }^{\text {a }}$ Malek T. Maghsoodlou, ${ }^{\text {a, }}$ Sayyed M. Habibi-Khorassani, ${ }^{\text {a }}$ Ghasem \\ Marandi, ${ }^{a}$ Khatereh Khandan-Barani, ${ }^{a}$ Morteza Ziyaadini, ${ }^{a}$ and Ali Aminkhani ${ }^{\text {b }}$ \\ ${ }^{a}$ Department of Chemistry, University of Sistan and Balouchestan, P. O. Box 98135-674, \\ Zahedan, Iran \\ ${ }^{b}$ Faculty of Science, Islamic Azad University of Khoy, Khoy, Iran \\ E-mail: mtmaghsoodlou@yahoo.com
}

\begin{abstract}
The reactive 1:1 intermediate is trapped from reaction between alkyl isocyanides and activated acetylenic esters by di-(2-pyridyl) ketone or 2-pyridinecarboxaldehyde. An effective and one-pot route is presented to synthesize novel iminolactones and 2-aminofurans.
\end{abstract}

Keywords: Three component condensation, alkyl isocyanides, acetylenic esters, di-(2-pyridyl) ketone, 2-pyridinecarboxaldehyde

\section{Introduction}

Multi-component processes are at a premium for the achievement of high levels of diversity and brevity, as they allow three or more simple and flexible building blocks to be combined in practical, one-pot operations. ${ }^{1-3}$ A few years ago it was reported, ${ }^{4}$ that the reaction between alkyl isocyanides and 3-benzylidene-2,4-pentanedione was a convenient route to prepare densely functionalized furans. Indeed, 2-aminofurans are quite $\operatorname{rare}^{5}$ and, according to the previous literature, rather difficult to prepare. ${ }^{6}$

Recently, multicomponent reactions (MCRs) have emerged as a highly valuable synthetic tool in the context of modern drug discovery. 5-Imino-2,5-dihydrofurans are potentially amenable to a number of synthetic transformation For example, they can be easily hydrolyzed to $\alpha, \beta$-unsaturated $\gamma$-lactones, a structural motif present in a number of bioactive natural products such as chlorothricolide, kijanolide, and tetranolide. ${ }^{7}$ It has been shown that alkyl or aryl isocyanides add to dialkyl acetylenedicarboxylates to generate zwitterionic species, which serve as intermediates in many different reaction. ${ }^{8-14}$ 
In previous works, the highly reactive 1:1 adduct, was trapped by carbonyl compounds to form 2,5-dihydro-2-imino- or 2-amino-furan derivatives in excellent yields. These reactions have been the subject of detailed investigation by a number of research groups. ${ }^{15-17}$ In view of our general interest in multicomponent reactions involving zwitterionic species, we examined the reaction of alkyl isocyanides $\mathbf{1}$ or $\mathbf{5}$ and dialkyl acetylenedicarboxylates $\mathbf{2}$ with di-(2-pyridyl) ketone 3 or 2-pyridinecarboxaldehyde 6 (Scheme 1).

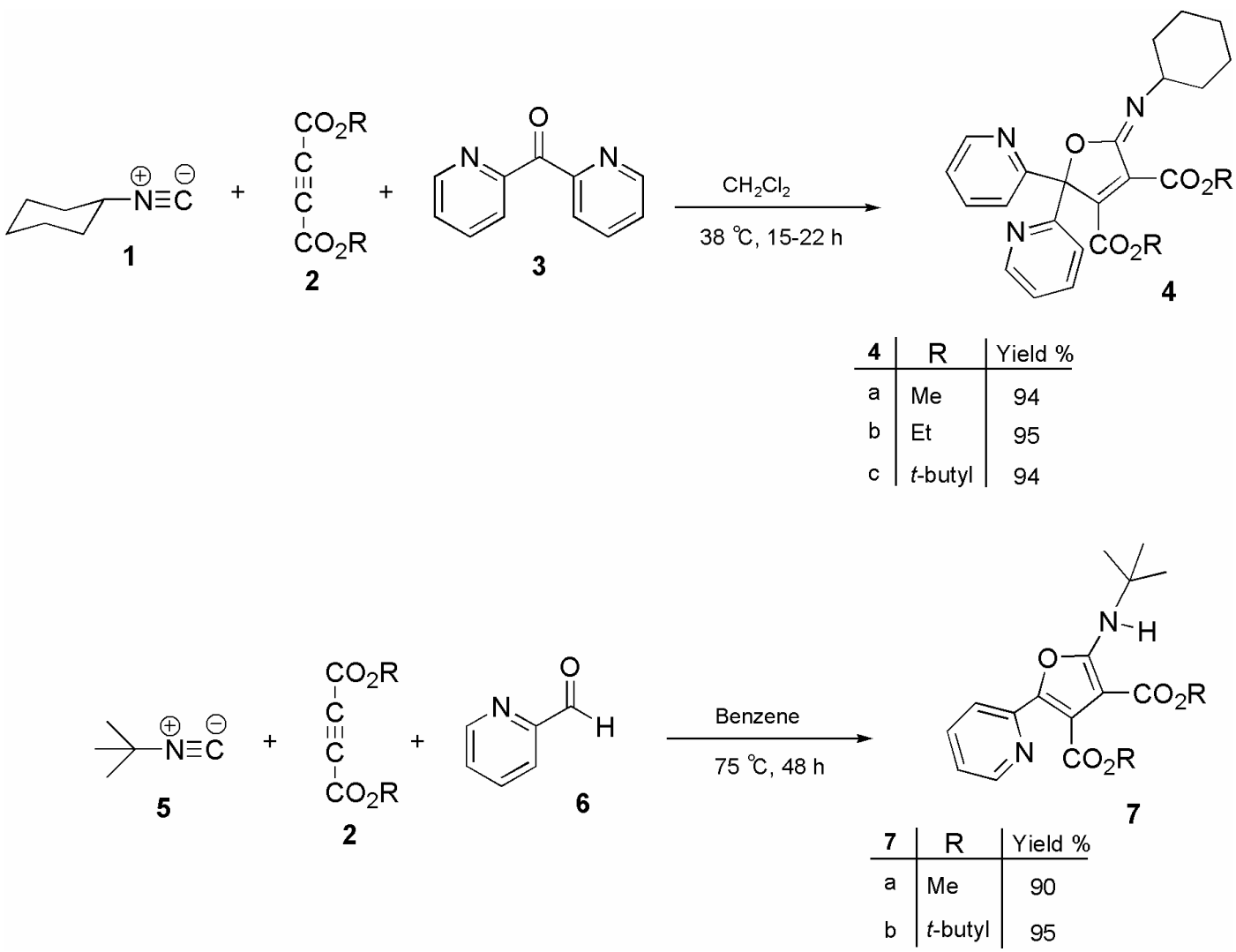

\section{Scheme 1}

\section{Results and Discussion}

The reaction of alkyl isocyanides $\mathbf{1}$ or $\mathbf{5}$ with dialkyl acetylenedicarboxylates $\mathbf{2}$ in the presence of pyridine-containing carbonyl compounds $\mathbf{3}$ or $\mathbf{6}$ leads to the stable products $\mathbf{4 a - c}$ or $\mathbf{7 a}, \mathbf{b}$ in excellent yields. A mechanistic rationale could be proposed for the formation of iminolactones or 2-aminofurans is shown (Scheme 2). The 1:1 zwitterionic intermediate which adds to the di-(2pyridyl) ketone leading to a dipolar species, cyclization of the latter leads to the iminolactone derivatives 4a-c, in the presence of 2-pyridinecarboxaldehyde, furan forms. This reaction, undergoes a [1,5]- hydrogen shift to yield the aminofuran derivatives $7 \mathbf{a}, \mathbf{b}$. 
It is conceivable that these multicomponent reactions will be applicable to the synthesis of heterocyclic rings with high hindrance. Products $\mathbf{4 a - c}$ and $\mathbf{7 a}, \mathbf{b}$ are stable solids which structures were deduced from their IR, ${ }^{1} \mathrm{H}$-, and ${ }^{13} \mathrm{C}$ - NMR, Mass spectral data and elemental analysis. The ${ }^{1} \mathrm{H}$ - NMR spectrum of compound $\mathbf{4 a}$ exhibited two singlet sharp lines, readily recognizable as arising from carbomethoxy groups (at $\delta 3.82$ and 3.93) ppm. The ${ }^{13} \mathrm{C}$ - NMR spectrum of 4a showed seventeen distinct resonances in a good agreement with iminolactone structure. The characteristic signals resulting from the quaternary carbon and $\mathrm{C}=\mathrm{N}$ group of iminolactone were discernible at ( $\delta 94.73$ and 155.84$)$ ppm respectively in the ${ }^{13} \mathrm{C}$ - NMR spectrum. Partial assignments of these resonances are given in the experimental data.

The ${ }^{1} \mathrm{H}$ - NMR spectrum of compound 7a exhibited three single sharp lines, readily recognizable as arising from tert-butyl $(\delta 1.52)$ and two carbomethoxy groups $(\delta 3.79$ and 3.98) ppm and NH proton resonated at $\left(\delta\right.$ 6.92) ppm supporting the IR absorption at $3335 \mathrm{~cm}^{-1}$. The ${ }^{13} \mathrm{C}$ - NMR spectrum of $7 \mathbf{a}$ showed fifteen distinct resonances in an agreement with proposed structure. Signals resulting from two ester carbonyl were discernible at ( $\delta 164.26$ and 164.91) ppm in the ${ }^{13} \mathrm{C}$ NMR spectrum. The mass spectra of these compounds $\mathbf{7 a}, \mathbf{b}$ displayed molecular ion peaks at appropriate $m / z$ values. The ${ }^{1} \mathrm{H}$ - and ${ }^{13} \mathrm{C}$ - NMR spectra of $7 \mathbf{b}$ are similar to $7 \mathbf{a}$ with the exception of the carboalkoxy groups.
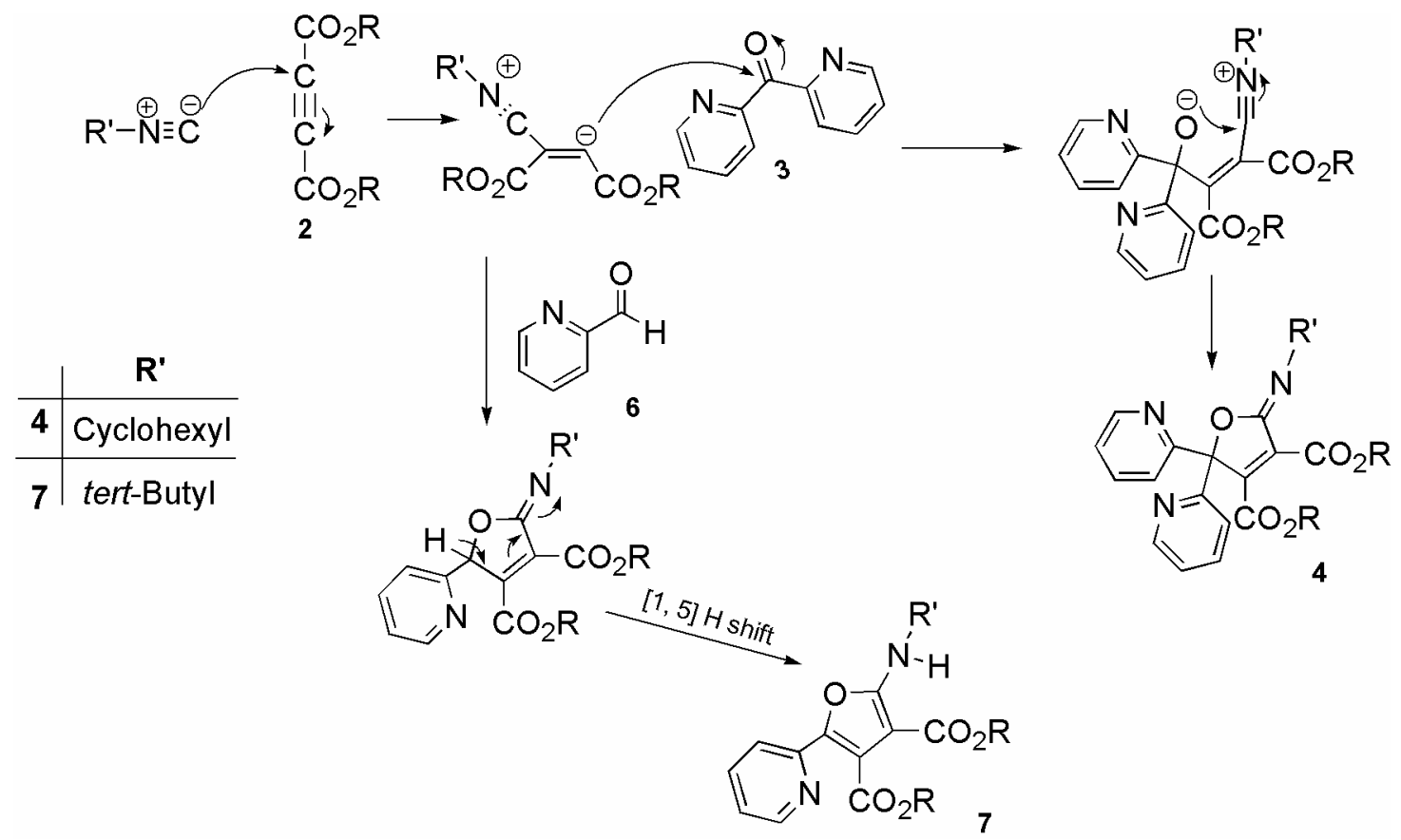

\section{Scheme 2}

In conclusion, we describe here, the reaction of alkyl isocyanides with activated acetylenes in the presence of pyridine-containing carbonyl compounds that leads to the one-pot and important synthesis of highly hindered and functionalized iminolactone or 2-aminofuran derivatives. The 
substances can be mixed without any activation or modification in these reactions, which in the view of experimental it is an important advantages.

\section{Experimental Section}

General Procedures. tert-Butyl- and cyclohexyl isocyanides, dialkyl acetylenedicarboxylates, di-(2-pyridyl) ketone and 2-pyridinecarboxaldehyde were purchased from Fluka and Aldrich, respectively, and used without further purification. Melting points and IR spectra were measured on an Electrothermal 9100 apparatus and a Shimadzu IR-470 spectrometer, respectively. Elemental analysis for $\mathrm{C}, \mathrm{H}$ and $\mathrm{N}$ were performed using a Heraeus $\mathrm{CHN}-\mathrm{O}$-Rapid analyzer. The ${ }^{1} \mathrm{H}$ - and ${ }^{13} \mathrm{C}$ NMR spectra were measured with a Bruker DRX-300 AVANCE instrument with $\mathrm{CDCl}_{3}$ as solvent at 300.1 and $75.5 \mathrm{MHz}$, respectively. Mass spectra were recorded on a Shimadzu GC/MS QP 1100 EX mass spectrometer operating at an ionization potential of $70 \mathrm{eV}$.

\section{General experimental procedure (exemplified by 4a)}

The process for the preparation of $\mathbf{4 a}$ is described as an example. The solution of cyclohexyl isocyanide $(0.131 \mathrm{~g}$ or $1.2 \mathrm{mmol})$ in $3 \mathrm{~mL}$ of $\mathrm{CH}_{2} \mathrm{Cl}_{2}$ solvent was slowly added dropwise, to the mixture of di-(2-pyridyl) ketone $(0.184 \mathrm{~g}$ or $1 \mathrm{mmol})$ and DMAD $(0.171 \mathrm{~g}$ or $1.2 \mathrm{mmol})$ in 20 $\mathrm{mL}$ of $\mathrm{CH}_{2} \mathrm{Cl}_{2}$ solvent at room temperature for 3 minutes. After the complete addition, the solution was refluxed at $38^{\circ} \mathrm{C}$ for 16 hours. Then, the solvent was removed under reduced pressure, and the solid residual washed with cold diethyl ether $(2 \times 5 \mathrm{~mL})$ and the product $(\mathbf{4 a})$ was obtained as a brown powder.

Dimethyl 5-(cyclohexylimino)-2,5-dihydro-2,2-di-(2-pyridyl)-3,4-furandicarboxylate (4a). Brown powder; yield $0.41 \mathrm{~g}(94 \%), \mathrm{mp} 156-159{ }^{\circ} \mathrm{C}$, IR $(\mathrm{KBr})\left(v_{\max }, \mathrm{cm}^{-1}\right)$ : 1739 and 1714 $(2 \mathrm{C}=\mathrm{O}), 1680(\mathrm{C}=\mathrm{N}) .{ }^{1} \mathrm{H}-\mathrm{NMR}\left(300.1 \mathrm{MHz}, \mathrm{CDCl}_{3}\right): \delta_{\mathrm{H}} 1.28-2.20\left(10 \mathrm{H}, \mathrm{m}, 5 \mathrm{CH}_{2}\right.$ of cyclohexyl), $3.53(1 \mathrm{H}, \mathrm{m}, \mathrm{NCH}), 3.82$ and $3.93\left(6 \mathrm{H}, 2 \mathrm{~s}, 2 \mathrm{CO}_{2} \mathrm{CH}_{3}\right), 7.33-8.62(\mathrm{~m}, 8 \mathrm{CH}) .{ }^{13} \mathrm{C}$ NMR (75.5 MHz, $\left.\mathrm{CDCl}_{3}\right): \delta_{\mathrm{C}} 24.87,25.64,33.11\left(5 \mathrm{CH}_{2}\right.$ of cyclohexyl), 52.63 and 53.02 (2 $\left.\mathrm{OCH}_{3}\right), 56.66\left(\mathrm{NCH}\right.$ of cyclohexyl), $94.73\left(\mathrm{C}_{\text {quaternary }}\right), 122.58,123.45,124.03,136.70,137.11$, 146.17, $148.93\left(\mathrm{C}=\mathrm{C}_{\text {iminolactone ring }}\right.$ and $\left.\mathrm{C}_{\text {arom }}\right), 155.84\left(\mathrm{C}=\mathrm{N}_{\text {imine }}\right), 157.64$ and $162.77(2 \mathrm{C}=\mathrm{O})$. MS (m/z, \%): $435\left(\mathrm{M}^{+}, 2\right), 404$ (4), 352 (1), 338 (32), 311 (44), 279 (100), 221 (15), 192 (23), 106 (13), 78 (62), 55 (53), 41 (56). Anal. Calcd for $\mathrm{C}_{24} \mathrm{H}_{25} \mathrm{~N}_{3} \mathrm{O}_{5}$ (435): C, 66.21; H, 5.75; N, 9.66\%; Found: C, 65.38; H, 5.57; N, 9.81\%.

Diethyl 5-(cyclohexylimino)-2,5-dihydro-2,2-di-(2-pyridyl)-3,4-furandicarboxylate (4b). Grey crystals; yield $0.44 \mathrm{~g}(95 \%), \mathrm{mp} 126-129{ }^{\circ} \mathrm{C}$, IR (KBr) $\left(v_{\max }, \mathrm{cm}^{-1}\right): 1741$ and $1716(2$ $\mathrm{C}=\mathrm{O}), 1677(\mathrm{C}=\mathrm{N}) .{ }^{1} \mathrm{H}-\mathrm{NMR}\left(300.1 \mathrm{MHz}, \mathrm{CDCl}_{3}\right): \delta \mathrm{H} 1.14-1.81\left(10 \mathrm{H}, \mathrm{m}, 5 \mathrm{CH}_{2}\right.$ of cyclohexyl), $1.17\left(3 \mathrm{H}, \mathrm{t}, \mathrm{J}=7.1 \mathrm{~Hz}, \mathrm{OCH}_{2} \mathrm{CH}_{3}\right), 1.35\left(3 \mathrm{H}, \mathrm{t}, \mathrm{J}=7.1 \mathrm{~Hz}, \mathrm{OCH}_{2} \mathrm{CH}_{3}\right), 3.72(1 \mathrm{H}, \mathrm{m}$, $\mathrm{NCH}), 4.21\left(2 \mathrm{H}, \mathrm{q}, \mathrm{J}=7.1 \mathrm{~Hz}, \mathrm{OCH}_{2} \mathrm{CH}_{3}\right), 4.37\left(2 \mathrm{H}, \mathrm{q}, \mathrm{J}=7.1 \mathrm{~Hz}, \mathrm{OCH}_{2} \mathrm{CH}_{3}\right), 7.28-8.57$ (m, 8 $\mathrm{CH}) .{ }^{13} \mathrm{C}$ - NMR $\left(75.5 \mathrm{MHz}, \mathrm{CDCl}_{3}\right): \delta_{\mathrm{C}} 13.70$ and $13.99\left(2 \mathrm{CH}_{3}\right), 24.08,24.82$ and $31.02\left(5 \mathrm{CH}_{2}\right.$ of cyclohexyl), $56.49\left(\mathrm{NCH}\right.$ of cyclohexyl), 62.02 and $62.28\left(2 \mathrm{OCH}_{2}\right), 91.46\left(\mathrm{C}_{\text {quaternary }}\right)$, 
122.69, 123.32, 123.89, 136.61, 137.02, 148.81, $149.08\left(\mathrm{C}=\mathrm{C}_{\text {iminolactone ring }}\right.$ and $\left.\mathrm{C}_{\text {arom }}\right), 155.47$ $\left(\mathrm{C}=\mathrm{N}_{\text {imine }}\right), 159.84$ and $161.45(2 \mathrm{C}=\mathrm{O}) . \mathrm{MS}(\mathrm{m} / \mathrm{z}, \%): 463\left(\mathrm{M}^{+}, 1\right), 418(6), 390(7), 366(37), 339$ (53), 293 (100), 221 (38), 193 (44), 106 (11), 78 (49), 55 (38), 41 (35). Anal. Calcd for $\mathrm{C}_{26} \mathrm{H}_{29} \mathrm{~N}_{3} \mathrm{O}_{5}$ (463): C, 67.39; H, 6.26; N, 9.07\%; Found: C, 65.24; H, 6.39; N, 8.84\%.

Di-tert-butyl 5-(cyclohexylimino)-2,5-dihydro-2,2-di-(2-pyridyl)-3,4-furandicarboxylate

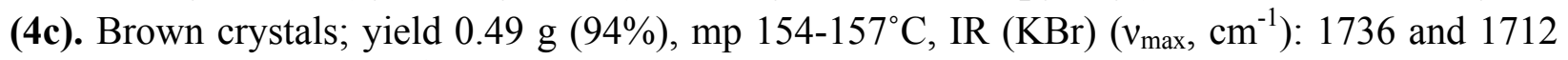
$(2 \mathrm{C}=\mathrm{O}), 1683(\mathrm{C}=\mathrm{N}) .{ }^{1} \mathrm{H}-\mathrm{NMR}\left(300.1 \mathrm{MHz}, \mathrm{CDCl}_{3}\right): \delta_{\mathrm{H}} 1.27-1.79\left(10 \mathrm{H}, \mathrm{m}, 5 \mathrm{CH}_{2}\right.$ of cyclohexyl), 1.35 and $1.60\left(18 \mathrm{H}, 2 \mathrm{~s}, 2 \mathrm{OCMe}_{3}\right), 3.75(1 \mathrm{H}, \mathrm{m}, \mathrm{NCH}), 7.29(2 \mathrm{H}, \mathrm{m}, 2 \mathrm{CH}), 7.36$ $(2 \mathrm{H}, \mathrm{t}, J=7.8 \mathrm{~Hz}, 2 \mathrm{CH}), 7.75\left(2 \mathrm{H}, \mathrm{dt}, J_{1}=7.8 \mathrm{~Hz}, J_{2}=1.0 \mathrm{~Hz}, 2 \mathrm{CH}\right), 8.56(2 \mathrm{H}, \mathrm{m}, 2 \mathrm{CH}) .{ }^{13} \mathrm{C}-$ NMR (75.5 MHz, $\left.\mathrm{CDCl}_{3}\right): \delta_{\mathrm{C}} 24.55,25.87,33.37$ (5 $\mathrm{CH}_{2}$ of cyclohexyl), 27.76 and 28.08 (2 $\left.\mathrm{CMe}_{3}\right), 55.75\left(\mathrm{NCH}\right.$ of cyclohexyl), 82.26 and $83.12\left(2 \mathrm{OCMe}_{3}\right), 94.37\left(\mathrm{C}_{\text {quaternary }}\right), 122.45$, $123.04,123.83,136.41,136.78,148.41,148.47\left(\mathrm{C}=\mathrm{C}_{\text {iminolactone ring }}\right.$ and $\left.\mathrm{C}_{\text {arom }}\right), 158.33\left(\mathrm{C}=\mathrm{N}_{\text {imine }}\right)$, 160.70 and $160.95(2 \mathrm{C}=\mathrm{O})$. MS $(\mathrm{m} / \mathrm{z}, \%): 521\left(\mathrm{M}^{+}+2,14\right), 520\left(\mathrm{M}^{+}+1,37\right), 519\left(\mathrm{M}^{+}, 4\right), 464(2)$, 446 (3), 418 (4), 318 (14), 283 (57), 265 (48), 238 (25), 221 (36), 193 (39), 106 (5), 57 (100), 41 (65). Anal. Calcd for $\mathrm{C}_{30} \mathrm{H}_{37} \mathrm{~N}_{3} \mathrm{O}_{5}$ (519): C, 69.36; H, 7.13; N, 8.09\%; Found: C, 66.16; H, 7.31; N, $8.26 \%$.

\section{General procedure (exemplified by 7 a)}

The preparation of $\mathbf{7 a}$ is described as an example. To the solution of 2-pyridinecarboxaldehyde $(0.107 \mathrm{~g}$ or $1 \mathrm{mmol})$ and DMAD $(0.171 \mathrm{~g}$ or $1.2 \mathrm{mmol})$ in $20 \mathrm{~mL}$ of benzene solvent, was slowly added, dropwise, a mixture of tert-butyl isocyanide $(0.100 \mathrm{~g}$ or $1.2 \mathrm{mmol})$ in $3 \mathrm{~mL}$ of benzene. To this mixture was allowed to stand for at room temperature for $3 \mathrm{~min}$, then the reaction mixture was refluxed at $75^{\circ} \mathrm{C}$ for $48 \mathrm{~h}$. The solvent was removed under reduced pressure, and the solid product washed with cold diethyl ether and n-hexane with $1: 3$ ratios $(2 \times 3 \mathrm{~mL})$ and the product (7a) was obtained as a brown powder.

Dimethyl 2-(tert-butylamino)-5-(2-pyridyl)-3,4-furandicarboxylate (7a). Brown powder; yield $0.30 \mathrm{~g}(90 \%), \mathrm{mp} \mathrm{83-86}{ }^{\circ} \mathrm{C}$, IR (KBr) $\left(v_{\max }, \mathrm{cm}^{-1}\right): 3335(\mathrm{~N}-\mathrm{H}), 1738$ and $1677(2 \mathrm{C}=\mathrm{O})$. ${ }^{1} \mathrm{H}-\mathrm{NMR}\left(300.1 \mathrm{MHz}, \mathrm{CDCl}_{3}\right): \delta_{\mathrm{H}} 1.52(9 \mathrm{H}, \mathrm{s}, \mathrm{NCMe}), 3.79$ and $3.98\left(6 \mathrm{H}, 2 \mathrm{~s}, 2 \mathrm{OCH}_{3}\right), 6.92$ $(1 \mathrm{H}, \mathrm{s}, \mathrm{NH}), 7.08\left(1 \mathrm{H}, \mathrm{dd}, J_{1}=5.0 \mathrm{~Hz}, J_{2}=0.8 \mathrm{~Hz}, \mathrm{Ar}-\mathrm{H}\right), 7.44(1 \mathrm{H}, \mathrm{d}, J=7.9 \mathrm{~Hz}, \mathrm{Ar}-\mathrm{H}), 7.68$ $\left(1 \mathrm{H}, \mathrm{dt}, J_{1}=7.8 \mathrm{~Hz}, J_{2}=1.7 \mathrm{~Hz}, \mathrm{Ar}-\mathrm{H}\right), 8.52(1 \mathrm{H}, \mathrm{d}, J=4.4 \mathrm{~Hz}, \mathrm{Ar}-\mathrm{H}) .{ }^{13} \mathrm{C}-\mathrm{NMR}(75.5 \mathrm{MHz}$, $\left.\mathrm{CDCl}_{3}\right): \delta_{\mathrm{C}} 29.80\left(\mathrm{NCMe}_{3}\right), 51.64\left(\mathrm{NCMe}_{3}\right), 53.25$ and $53.96(2 \mathrm{OMe}), 90.46,119.35,120.85$, 121.37, 134.10, 140.81, 145.50, 150.01 and $162.51\left(\mathrm{C}=\mathrm{C}_{\text {aminofuran ring }}\right.$ and $\left.\mathrm{C}_{\text {arom }}\right), 164.26$ and $164.91(2 \mathrm{C}=\mathrm{O})$. MS (m/z, \%): $334\left(\mathrm{M}^{+}+2,2\right), 333\left(\mathrm{M}^{+}+1,7\right), 332\left(\mathrm{M}^{+}, 35\right), 301$ (3), $276(45)$, 261 (4), 244 (13), 212 (62), 184 (37), 106 (33), 78 (100), 57 (91), 41 (96). Anal. Calcd for $\mathrm{C}_{17} \mathrm{H}_{20} \mathrm{~N}_{2} \mathrm{O}_{5}$ (332): C, 61.45; H, 6.02; N, 8.43\%; Found: C, 63.45; H, 6.28; N, 8.31\%

Di-tert-butyl 2-(tert-butylamino)-5-(2-pyridyl)-3,4-furandicarboxylate (7b). White powder; yield $0.40 \mathrm{~g}(95 \%), \mathrm{mp} 123-125^{\circ} \mathrm{C}$, IR $(\mathrm{KBr})\left(v_{\text {max. }}, \mathrm{cm}^{-1}\right): 3323(\mathrm{~N}-\mathrm{H}), 1714$ and $1673(2 \mathrm{C}=\mathrm{O})$. ${ }^{1} \mathrm{H}-\mathrm{NMR}\left(300.1 \mathrm{MHz}, \mathrm{CDCl}_{3}\right): \delta_{\mathrm{H}} 1.50(9 \mathrm{H}, \mathrm{s}, \mathrm{NCMe}), 1.56$ and $1.64\left(18 \mathrm{H}, 2 \mathrm{~s}, 2 \mathrm{OCMe}_{3}\right)$, $7.01(1 \mathrm{H}, \mathrm{s}, \mathrm{N} H), 7.05\left(1 \mathrm{H}, \mathrm{dd}, J_{1}=4.8 \mathrm{~Hz}, J_{2}=0.8 \mathrm{~Hz}, \operatorname{Ar} H\right), 7.42(1 \mathrm{H}, \mathrm{d}, J=8.0 \mathrm{~Hz}, \operatorname{Ar} H), 7.65$ 
$\left(1 \mathrm{H}, \mathrm{dt}, J_{1}=8.0 \mathrm{~Hz}, J_{2}=1.4 \mathrm{~Hz}, \operatorname{Ar} H\right), 8.48(1 \mathrm{H}, \mathrm{d}, J=4.8 \mathrm{~Hz}, \operatorname{Ar} H) .{ }^{13} \mathrm{C}-\mathrm{NMR}(75.5 \mathrm{MHz}$, $\left.\mathrm{CDCl}_{3}\right): \delta_{\mathrm{C}} 28.33$ and $28.73(2 \mathrm{xOCMe}), 29.16\left(\mathrm{NCMe}_{3}\right), 52.52\left(\mathrm{NCMe}_{3}\right), 80.69$ and 82.05 $(2 \mathrm{xOCMe}), \quad 90.25, \quad 117.35, \quad 118.53,120.74, \quad 136.25,139.11,148.26,148.90,161.52$ $\left(\mathrm{C}=\mathrm{C}_{\text {aminofuran ring }}\right.$ and $\left.\mathrm{C}_{\text {arom }}\right), 163.70$ and $164.52(2 \mathrm{C}=\mathrm{O})$. $\mathrm{MS}(\mathrm{m} / \mathrm{z}, \%): 417\left(\mathrm{M}^{+}+1,6\right), 416\left(\mathrm{M}^{+}\right.$, 17), 360 (10), 343 (2), 304 (42), 287 (6), 260 (13), 212 (9), 204 (93), 186 (54), 160 (40), 57 (100), 41 (63). Anal. Calcd for $\mathrm{C}_{23} \mathrm{H}_{32} \mathrm{~N}_{2} \mathrm{O}_{5}$ (416): C, 66.35; H, 7.69; N, 6.73\%; Found: C, 64.30; H, 7.66; N, 6.39\%.

\section{Acknowledgements}

We gratefully acknowledge financial support from the Research Council of University of Sistan and Balouchestan.

\section{References}

1. Dömling, A.; Herdtweck, E.; Heck, S. Tetrahedron Lett. 2006, 47, 1745.

2. Nair, V.; Sreekanth, A. R.; Abhilash, N. P.; Nair-Biju, A. T.; Varma, L.; Viji, S.; Mathew, S. Arkivoc 2005, (xi), 178.

3. Sapi, J.; Laronze, J. Y. Arkivoc 2004, (vii), 208.

4. Yavari, I.; Shaabani, A.; Maghsoodlou, M. T. Monatsh. Chem. 1997, 128, 697.

5. (a) John, I. G.; Radom, L. J. Am. Chem. Soc. 1978, 100, 3981. (b) Lythgoe, D. J.; McClenaghan, I.; Ramsden, C. A. J. Heterocycl. Chem. 1993, 30, 113.

6. Quai, M.; Frattini, S.; Vendrame, U.; Mondoni, M.; Dossena, S.; Cereda, E. Tetrahedron Lett. 2004, 45, 1413.

7. (a) Roush, W. R.; Reily, M. L.; Koyama, K.; Brown, B .B. J. Org. Chem. 1997, 62, 8708. (b) Roush, W. R.; Sciotti, R. J. Am. Chem. Soc. 1998, 120, 7411.

8. Nair, V.; Vinod, A. U. Chem. Commun. 2000, 1019.

9. (a) Yavari, I.; Esmaili, A. A.; Asghari, S.; Bijanzadeh, H. R. J. Chem. Res. (S). 1999, 368. (b) Yavari, I.; Hazeri, N.; Maghsoodlou, M. T.; Zabarjadshiraz, N. Monatsh. Chem. 2001, 132, 683.

10. (a) Shaabani, A.; Teimouri, M. B.; Mirzaei, P.; Bijanzadeh, H. R. J. Chem. Res. (S). 2003, 82. (b) Shaabani, A.; Teimouri, M. B.; Arab-Ameri, S. Tetrahedron Lett. 2004, 45, 8409.

11. Maghsoodlou, M. T.; Yavari, I.; Nassiri, F.; Djahaniani, H.; Razmjoo, Z. Monatsh. Chem. 2003, 134, 1585.

12. Nair, V.; Rajesh, C.; Vinod, A. U.; Bindu, S.; Sreekanth, A. R.; Mathen, J. S.; Balagopal, L. Acc. Chem. Res. 2003, 36, 899.

13. Yavari, I.; Maghsoodlou, M. T. J. Chem. Res. (S) 1998, 386. 
14. Maghsoodlou, M. T.; Hazeri, N.; Navvabian, H.; Razmjoo, Z.; Marandi, G. J. Chem. Res. 2005, 401.

15. Hazeri, N.; Maghsoodlou, M. T.; Habibi-Khorasani, S. M.; Ziyaadini, M.; Marandi, G.; Khandan-Barani, K.; Bijanzadeh, H. R. Arkivoc 2007, (xiii), 34.

16. Nair, V.; Vinod, A. U.; Somarajan Nair, J.; Sreekanth, A. R.; Rath, N. P. Tetrahedron Lett. 2000, 41, 6675 .

17. Maghsoodlou, M. T.; Hazeri, N.; Habibi-Khorasani, S. M.; Marandi, G.; Nassiri, M. Synth. Commun. 2005, 35, 2771. 